\title{
The impact of embryo binucleation depends upon its origin
}

\author{
Lia Mara Gomes Paim ${ }^{1}$ and Greg FitzHarris ${ }^{1,2}$ \\ ${ }^{1}$ Centre de Recherche du Centre Hospitalier de I'Université de Montréal, Montréal, Québec, Canada and \\ ${ }^{2}$ Département d'Obstétrique-Gynécologie, Université de Montréal, Montréal, Québec, Canada
}

Correspondence should be addressed to G FitzHarris; Email: greg.fitzharris@umontreal.ca

\begin{abstract}
Summary
Preimplantation embryos frequently contain binucleated cells, but reports differ as to whether binucleation affects development and whether such embryos should be used clinically. In this Point Of View article, we propose a possible explanation for this disparity: binucleation can arise by distinct routes, one that produces healthy blastomeres and one that directly threatens embryo viability. Reproduction (2020) $\mathbf{1 6 0} \mathrm{V} 1-\mathrm{V} 4$
\end{abstract}

The success of in vitro fertilisation cycles relies heavily on the choice of the most suitable embryo to be transferred to patients. Despite the emergence in recent years of a myriad of exotic means of embryo testing, embryo selection remains mainly based on morphological features observable under the light microscope, such as stage appropriate cell numbers, cytoplasmic fragmentation patterns, and nucleus configuration. Binucleation is the scenario wherein a blastomere within an embryo contains two evenly sized nuclei and is an example of a feature of nuclear morphology that can be seen under a microscope. Note that, in this Point of View article, we refer principally to truly binucleated embryos and not to other types of nuclear aberration such as micronucleation, where cells contain an extra 'mini' nucleus, or multinucleation, where three or more nuclei are seen in a blastomere. Binucleation is a relatively common phenomenon in human embryos in in vitro fertilisation cycles, with frequencies ranging from 7 to $65 \%$ of embryos depending on the stage of development examined (Hardy et al. 1993, Meriano et al. 2004, Aguilar et al. 2016, Seikkula et al. 2018). Although it is perhaps intuitive that to have two nuclei in a given cell should be indicative of poor prognosis for the embryo, contrasting and perhaps puzzling results as to the developmental potential and clinical outcomes of these embryos have been reported. Some studies report that the presence of binucleated blastomeres causes decreased developmental potential and implantation rates (Hardy et al. 1993, Aguilar et al. 2016, Seikkula et al. 2018). On the other hand, perhaps surprisingly, other reports seem to suggest that embryos containing binucleated blastomeres have relatively normal developmental potential (Staessen \& Van Steirteghem 1998, Meriano et al. 2004). An explanation for the apparently divergent reports is not apparent, and thus the use of binucleation as a means of embryo selection is not widely implemented. Here, following recent mechanistic studies of cell divisions and nuclear morphology in mouse embryos, we propose that binucleation can originate by two mechanistically distinct routes, depending on whether it occurs at the 2-cell stage or at later stages of development, and that these two routes to binucleation have contrasting implications for embryo health that explain the apparent conflicting reports.

The origins of binucleation in mid-preimplantation development ( 16-cell stage) human embryos were studied by Hardy et al. (1993), who found that binucleated blastomeres at the 16-32 cell stage had double the amount of cytoplasm compared to mononucleated counterparts, and thus concluded that binucleated blastomeres arise as a result of a failure of cytokinesis, the final step of cell division. Although other routes to binucleation can be envisaged, such as cell fusion, cytokinesis failure is generally considered the most common route to binucleation in somatic cells, and thus the conclusions of Hardy et al. appear very likely. Cytokinesis failure (or indeed cell fusion) means that the resulting blastomere contains an entire duplicated genome complement (i.e. tetraploid). Consistent with this, complete tetraploidy has been described in spontaneous abortions (Genest et al. 1995), as well as in embryos undergoing preimplantation genetic screening (Bielanska et al. 2002), and the rare cases of tetraploid live-born infants display severe developmental abnormalities, with a lifespan limited to months (Stefanova et al. 2010), indicative that tetraploidy is indeed a feature of early embryo development and poses severe consequences for fertility and life. Recent mechanistic studies in mouse embryos have uncovered at least two reasons why tetraploid blastomeres in midpreimplantation development should jeopardise the likelihood of a successful pregnancy. First, it was shown 
that tetraploidy resulting from cytokinesis failure at the 4-8 cell division increases the rates of chromosome segregation errors and consequently favors the development of further aneuploidy (Paim \& FitzHarris 2019) (Fig. 1). Such errors could lead to the complex and chaotic karyotypes seen in embryo blastomeres which are widely associated with embryonic failure (Bielanska et al. 2002). Second, tetraploidy in early mouse embryos has been shown to reduce the number of cells available to form the inner cell mass within the blastocyst that forms the embryo proper (Eakin et al. 2005, LMG Paim and G FitzHarris, unpublished observations). Why this decrease in the number of cells takes place is not entirely clear, but it is likely that fewer but larger cells at the time of blastocoel formation reduce the availability of 'inner' cells to form the ICM. Thus, binucleation in later stage embryos likely occurs by cytokinesis failure and threatens embryo viability in at least two ways (Fig. 1).

In contrast, a recent examination of the first mitosis (1-cell to 2-cell transition) in mouse leads us to suggest that binucleation at the 2-cell stage in human embryos may arise by an entirely different means. Reichmann et al. (2018) demonstrated that each pronucleus in the mouse zygote originates two spatially separate parental genomes that initially remain individualised during the first mitotic division (Fig. 1). As the first mitosis progresses, two independent spindles are assembled (one for each parental genome), which eventually fuse to align the maternal and paternal genome before anaphase. Interestingly, experimentally induced failure to align the two zygotic spindles leads to the formation of perfectly binucleated 2-cell embryos (Reichmann
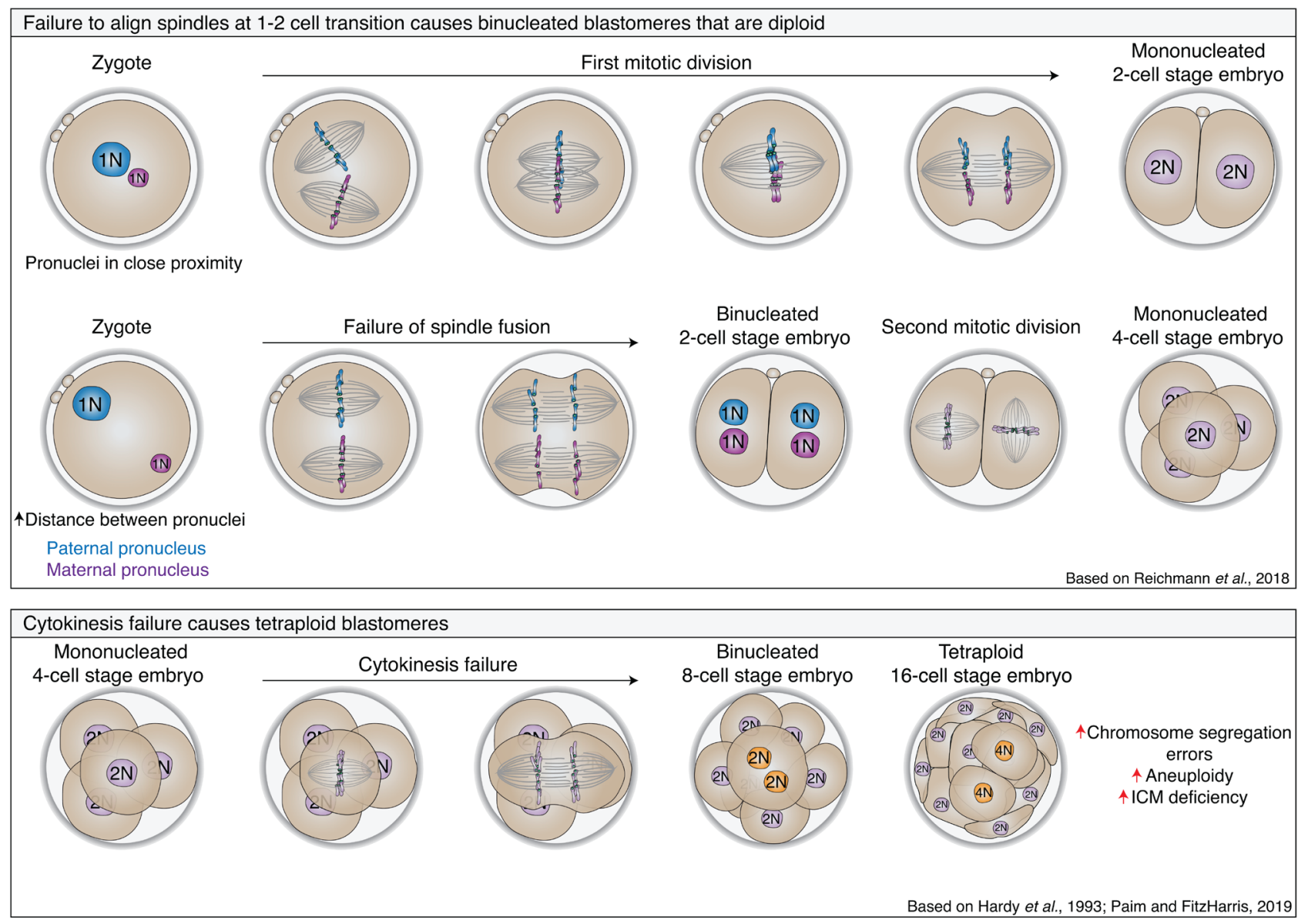

Figure 1 Two mechanistically distinct modes of binucleation might explain divergent clinical outcomes. Top panel: At the zygote stage, the paternal and maternal pronuclei are maintained physically separated due to the assembly of two individualized mitotic spindles. Further into the first mitotic division, the spindles fuse such that the parental genome is aligned in a single metaphase plate, allowing cell division to take place, and a diploid mononucleated 2-cell stage embryo is formed. Middle panel: In cases where the two parental pronuclei are too distant from each other, the two individualized spindles fail to fully fuse and cell division takes place without alignment of the parental genomes, resulting in a 2-cell stage embryo with two haploid nuclei. This scenario is likely not deleterious to development, since the resulting 2-cell embryo remains with a diploid genome complement. Bottom panel: Binucleation later in development more likely indicates cytokinesis failure or regression (which may be mistaken optically for cell fusion), which would lead to tetraploidy. This is followed by an increase in the levels of chromosomes segregation errors, allowing for the accumulation of highly aneuploid blastomeres that potentially lead to low implantation rates. 
et al. 2018) (Fig. 1). Although the implications of this were not explored, each nucleus would be haploid and thus each cell should logically be overall diploid. Since binucleated cells can assemble a single spindle and form mononucleated daughter cells (Paim \& FitzHarris 2019), resulting 4-cell embryos would be expected to be mononucleated, euploid (overall correct chromosome complement), and as such be identical to other 'normal' embryos that were never binucleated (Fig. 1). Thus, although it remains to be formally tested, we propose that binucleation at the 2-cell stage under these circumstances likely has minimal impact upon developmental potential.

To our knowledge, this explanation to the varied results of clinical binucleation studies mentioned has not previously been proposed. And while the tendency of many clinical studies to group binucleation in conjunction with other forms of nuclear aberration (micronucleation, multinucleation - which themselves very likely have severe consequences for embryo development (Vazquez-Diez et al. 2016)) muddies the issue in some cases, close examination of a few key papers lends some support for our proposal. Specifically, Staessen and Van Steirteghem (1998) showed that blastomeres karyotyped at the 3-8 cell stage from embryos previously scored as binucleated at the 2-cell stage are frequently diploid. This strongly supports the notion that the binucleated 2-cell blastomere can be diploid. Perhaps most intriguingly, one study that carefully distinguished binucleated embryos from multinucleated and mononucleated, and then by developmental stage, found that embryos displaying 2/4 binucleated blastomeres at the 4-cell stage never implanted, whereas implantation rates were normal in embryos binucleated at the 2-cell stage (Aguilar et al. 2016). The studies described previously (Staessen \& Van Steirteghem 1998, Bielanska et al. 2002) used karyotyping techniques now considered outdated such as FISH, and further analyses of the ploidy status of binucleated embryos using more reliable modern approaches are certainly warranted. Nevertheless, the findings described by Aguilar et al. (2016), Reichmann et al. (2018), and Paim and FitzHarris (2019) support the notion that binucleation at different stages of development might affect embryo health differently. Therefore, we hypothesise that the discordant conclusions on the impact of binucleation are a result of the different impacts of binucleation at different developmental stages; whereas binucleation in mid- to late-preimplantation development very likely indicates an error of chromosomal content, binucleation at 2-cell stage may not.

How and why cytokinesis failure comes about, whether a single binucleated/tetraploid blastomere is sufficient to significantly disrupt embryonic development at a given developmental stage, and to what extent embryos can tolerate binucleation/tetraploidy and still give rise to healthy live births, all remain unknown. However, the developments discussed here underscore the importance of foundational molecular research to understand and interpret the biological basis of phenomena observed in the clinic. Non-invasive means of selecting the best embryo(s) for transfer in the clinic remains one of the holy grails of reproductive medicine, and it is our hope that this re-interpretation of the impact of binucleation, paired with improvements in time-lapse microscopy, may ultimately lead to better paradigms and tools for embryo sorting. Essential in this will be further studies that carefully distinguish the developmental stage at which true binucleation occurs.

\section{Declaration of interest}

G FitzHarris is on the editorial board of Reproduction. G FitzHarris was not involved in the review or editorial process for this paper, on which he is listed as an author. L M Gomes Paim has nothing to disclose.

\section{Funding}

This work did not receive any specific grant from any funding agency in the public, commercial, or not-for-profit sector.

\section{Author contribution statement}

L M G P and G F wrote the manuscript. L M G P produced the figure.

\section{Acknowledgements}

The authors thank Drs Alexander Bruce and Jim Meriano for comments on the manuscript. Work in G F's lab is supported by grants from the Natural Sciences and Engineering Research Council of Canada, Fondation Jean-Louis Lévesque, Canadian Foundation for Innovation, and the Canadian Institutes of Health Research. L M G P is supported by a Fonds de Recherche du Québec - Santé Doctoral Scholarship.

\section{References}

Aguilar J, Rubio I, Munoz E, Pellicer A \& Meseguer M 2016 Study of nucleation status in the second cell cycle of human embryo and its impact on implantation rate. Fertility and Sterility 106 291.e2-299.e2. (https://doi.org/10.1016/j.fertnstert.2016.03.036)

Bielanska M, Tan SL \& Ao A 2002 Chromosomal mosaicism throughout human preimplantation development in vitro: incidence, type, and relevance to embryo outcome. Human Reproduction 17 413-419. (https://doi.org/10.1093/humrep/17.2.413)

Eakin GS, Hadjantonakis AK, Papaioannou VE \& Behringer RR 2005 Developmental potential and behavior of tetraploid cells in the mouse embryo. Developmental Biology 288 150-159. (https://doi. org/10.1016/j.ydbio.2005.09.028)

Genest DR, Roberts D, Boyd T \& Bieber FR 1995 Fetoplacental histology as a predictor of karyotype: a controlled study of spontaneous first trimester abortions. Human Pathology 26 201-209. (https://doi. org/10.1016/0046-8177(95)90038-1)

Hardy K, Winston RM \& Handyside AH 1993 Binucleate blastomeres in preimplantation human embryos in vitro: failure of cytokinesis during 
early cleavage. Journal of Reproduction and Fertility 98 549-558. (https://doi.org/10.1530/jrf.0.0980549)

Meriano J, Clark C, Cadesky K \& Laskin CA 2004 Binucleated and micronucleated blastomeres in embryos derived from human assisted reproduction cycles. Reproductive Biomedicine Online 9 511-520. (https://doi.org/10.1016/s1472-6483(10)61635-5)

Paim LMG \& Fitzharris G 2019 Tetraploidy causes chromosomal instability in acentriolar mouse embryos. Nature Communications 104834. (https://doi.org/10.1038/s41467-019-12772-8)

Reichmann J, Nijmeijer B, Hossain MJ, Eguren M, Schneider I, Politi AZ, Roberti MJ, Hufnagel L, Hiiragi T \& Ellenberg J 2018 Dual-spindle formation in zygotes keeps parental genomes apart in early mammalian embryos. Science 361 189-193. (https://doi.org/10.1126/science. aar7462)

Seikkula J, Oksjoki S, Hurme S, Mankonen H, Polo-Kantola P \& Jokimaa V 2018 Pregnancy and perinatal outcomes after transfer of binucleated or multinucleated frozen-thawed embryos: a case-control study. Reproductive Biomedicine Online 36 607-613. (https://doi. org/10.1016/j.rbmo.2018.02.003)

Staessen C \& Van Steirteghem A 1998 The genetic constitution of multinuclear blastomeres and their derivative daughter blastomeres.
Human Reproduction 13 1625-1631. (https://doi.org/10.1093/ humrep/13.6.1625)

Stefanova I, Jenderny J, Kaminsky E, Mannhardt A, Meinecke P, Grozdanova L \& Gillessen-Kaesbach G 2010 Mosaic and complete tetraploidy in live-born infants: two new patients and review of the literature. Clinical Dysmorphology 19 123-127. (https://doi.org/10.1097/ MCD.0b013e3283353877)

Vazquez-Diez C, Yamagata K, Trivedi S, Haverfield J \& Fitzharris G 2016 Micronucleus formation causes perpetual unilateral chromosome inheritance in mouse embryos. PNAS 113 626-631. (https://doi. org/10.1073/pnas.1517628112)

Received 2 April 2020

First decision 9 April 2020

Revised manuscript received 10 April 2020

Accepted 24 April 2020 